\section{PHYSICAL CHEMISTRY}

\section{Growing up bigger}

Angew. Chem. Int. Ed. 47, 4208-4210 (2008)

Which is smaller: hydrogen or deuterium? The standard answer is that deuterium $\left({ }^{2} \mathrm{H}\right)$ takes up less space than ${ }^{1} \mathrm{H}$ because its greater mass gives it a smaller vibration amplitude in the lowest-energy quantum state. But the veracity of this argument is temperature sensitive, say Jack Dunitz of ETH-Zurich in Switzerland and Richard Ibberson of the Rutherford Appleton Laboratory in Didcot, UK.

By taking careful measurements of the crystal structures of benzene and fully deuterated benzene at temperatures between 5 kelvin and 280 kelvin, they show that the volume of a molecule of the latter exceeds that of the former above about 170 kelvin. At such temperatures, the vibrations of the carbon-deuterium bonds include more of the higher-energy quantum states than those of C- ${ }^{1} \mathrm{H}$ bonds, which means that the deuterium atoms effectively occupy more space.

\section{GENETICS}

\section{Genes with bottle}

Nature Genet. doi:10.1038/ng.151 (2008)

Researchers have pinpointed a pair of gene variants that seem to protect their carriers against head and neck cancers linked to alcohol consumption. The variants are of genes that encode alcohol dehydrogenase (ADH) enzymes, which catalyse the breakdown of alcohols into aldehydes.

Paul Brennan at the International Agency for Research on Cancer in Lyon, France, and his colleagues analysed six ADH variants in 3,876 patients with head and neck cancers and 5,278 healthy people. A variant of each of the two genes $A D H 1 B$ and $A D H 7$ seemed to lower the risk of developing these cancers in alcohol drinkers, most dramatically in heavy drinkers. Carriers of the $A D H 1 B$ variant metabolize alcohol up to 100 times faster than non-carriers.

\section{GEOPHYSICS}

\section{The heat is on}

Earth Planet. Sci. Lett. doi:10.1016/ j.epsl.2008.03.031 (2008)

A planet's interior affects its climate through volcanoes spewing out greenhouse gases. Conversely, the climate can also affect the interior, according to calculations by Adrian Lenardic, of Rice University in Houston, Texas, and his co-workers.

They worked out that temperature increases at a terrestrial planet's surface could penetrate deep into the planet, rendering its mantle less viscous and eventually shutting down the movement of tectonic plates.

For a planet such as Earth, a sustained rise of 100 kelvin over a 10-million to 100 million-year timescale could be enough to destablize plate tectonics. The authors suggest that the carbon dioxide blanket in Venus's atmosphere (artist's impression, pictured) might help to explain why it appears to have a single, static plate.

\section{GENETICS}

\section{The sweet life}

Hum. Mol. Genet. doi:10.1093/hmg/ddn137 (2008) Different versions of the gene that encodes insulin-degrading enzyme (IDE) are associated with how long men - but not women - live, researchers have found.

Insulin metabolism had previously been linked to the lifespans of organisms commonly used in laboratory research. Jonathan Prince of

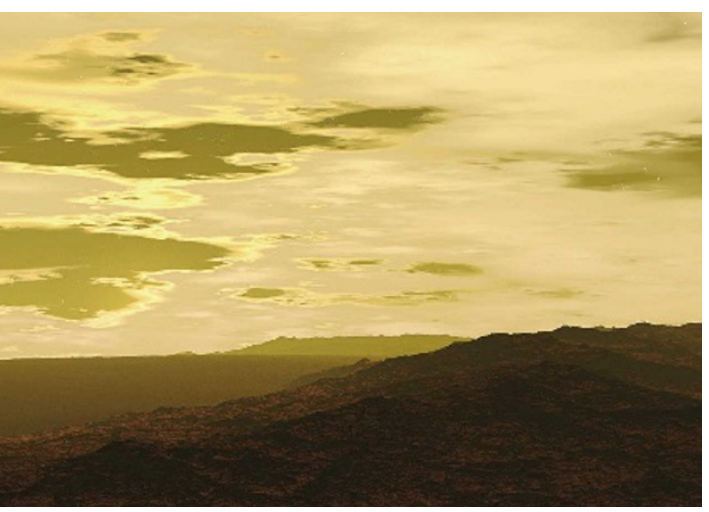

the Karolinska Institute in Stockholm, Sweden and his colleagues now report that human males with one copy of a certain version of IDE and one copy of a different version make higher than usual amounts of IDE RNA and produce more insulin when they fast. These men also tended to die younger than those carrying two copies of the IDE variant.

\section{MICROBIOLOGY Inner lives}

Science doi:10.1126/science.1155725 (2008) How particular to humans are the microbes that eek out a living in our intestines? To find out, Jeffrey Gordon of Washington University School of Medicine in St Louis, Missouri, and his colleagues compared particular genetic sequences from gut microbes found in the faeces of 106 mammals, some wild and others from American zoos.

Among the 60 species represented in this sample, carnivores tend to have a less diverse internal flora than omnivores, and omnivores less than hervibores. Modern humans support similar gut microbes to those of other omnivores - a surprise, maybe, given the importance of agriculture and cookery in human ecological history.

\section{JOURNAL CLUB}

\section{Nathan Wolfe \\ University of California, Los Angeles}

An epidemiologist points to a fifth sort of human malaria.

Malaria has plagued humans since the dawn of written history, and probably since long before that. These days, biologists understand tiny mechanistic details of the workings of one human malarial parasite, Plasmodium falciparum, but know surprisingly little about the others. As someone who studies how pandemics are born and die - and how they might one day be prevented - these holes in our knowledge seem striking to me.

Aside from $P$. falciparum - the cause of 'malignant' malaria parasitologists acknowledge three other human malaria parasites, $P$. vivax, $P$. ovale and $P$. malariae, each of which probably jumped from another primate host to humans independently. With so many malaria parasites plaguing other vertebrate species, however, and only basic diagnostic instruments available in most parts of the world, science could be missing new types of human malaria that have the potential to seed pandemics.

In a recent paper, Janet CoxSingh and her colleagues build on their earlier finding that humans can harbour a fifth malaria parasite, P. knowlesi, which was once thought to infect only Asian monkeys. The researchers detected $P$. knowlesi DNA in about one third of 1,014 malaria patients in Malaysia, showing that this parasite is common, deadly and almost always misidentified as P. malariae (J. Cox-Singh et al. Clin. Infect. Dis. 46, 165-171; 2008).
That an unknown animal pathogen can cause widespread human disease is reminiscent of some of the biggest scourges of the twentieth century: HIV and pandemic influenza. Reductionist, molecular approaches to tackling important plagues may be en vogue and a near necessity for grant funding, but I bet that an oldfashioned natural historian studying how infectious agents jump host species will be first to signal the coming of the next plague.

Discuss this paper at http://blogs. nature.com/nature/journalclub 
Jon Lundberg of the Karolinska Institute in Stockholm and his colleagues had set out to study the importance of natural gut bacteria in nitrate metabolism using mice bred to harbour no microorganisms. But when these germ-free mice were fed sodium nitrate, and nitrite ions then showed up in their blood, the team began hunting for enzymes to explain the result.

They recorded nitrate-reducing activity from xanthine oxidoreductase in the liver tissue of both rodents and humans, and found that $\mathrm{NO}_{2}^{-}$can be further reduced to NO. The pathway ramped up during an experiment in which the researchers clamped the abdominal aorta of rats - perhaps unsurprisingly, given that NO dilates blood vessels.

\section{NANOTECHNOLOGY}

\section{Sheet change}

Nanoletters doi:10.1021/nl0808132 (2008)

Researchers have worked out how to detect trace amounts of amyloid- $\beta$ protein that has undergone the conformational change seen in people with Alzheimer's disease.

Gerard Coté of Texas A\&M University in College Station and his co-workers built a nanofluidic device able to concentrate amyloid- $\beta$ proteins and gold nanoparticles at the entrance of a tiny channel by exploiting capillary flow. They then performed surfaceenhanced Raman spectroscopy on the concentrated amyloid- $\beta$ proteins, searching out any with a structure predominantly composed of $\beta$-sheets, a common folding arrangement.

This may allow doctors to test the cerebrospinal fluid of patients with cognitive decline and identify those who will go on to develop Alzheimer's disease, the authors say.

\section{CHEMICAL SENSING}

\section{Molecular mapping}

Angew. Chem. Int. Edn doi:10.1002/anie.200801516 (2008)

A family of molecular proton sensors that can be programmed to sit at specific distances from the surface of a membrane has been devised by Seiichi Uchiyama and Prasanna de Silva at Queen's University in Belfast, UK, and Kaoru Iwai at Nara Women's University in Japan.

These molecules have position-tuning groups that 'seek out' a local environment that matches their own compatibility with water. They thus distribute themselves at various distances along a radial coordinate of a membranous sphere called a micelle. The local proton concentration determines the intensity of emission from a fluorescent 'reporter' group on the sensor, whereas the asymmetry of local electronic-

charge polarity determines the emission wavelength.

All of these details can be mapped. This technique might one day provide clues about how biological surfaces and structures function.

\section{ZOOLOGY}

\section{Mid-ocean wanderer}

Biol. Lett. doi:10.1098/rsbl.2008.0147 (2008)

Basking sharks (Cetorhinus maximus; pictured below) thought to exist in discrete populations thousands of kilometres apart may in fact mix by migration, say scientists who tracked a female across the Atlantic.

Previous tagging experiments have shown

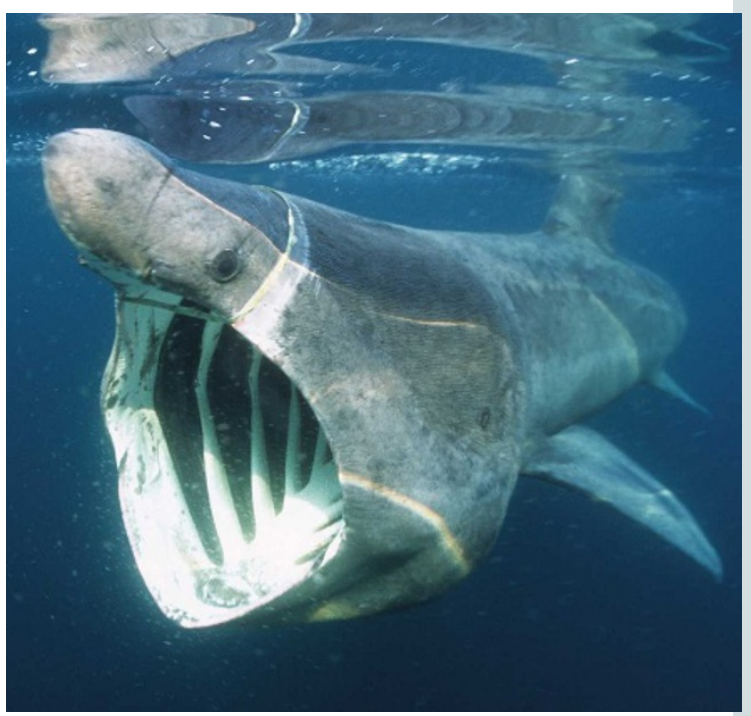

apparently distinct populations of basking sharks migrating south for winter at shallow depths along the continental shelf of Europe and the east coast of North America.

But a basking shark tagged by Mauvis Gore of Marine Conservation International in Newton, UK, and her colleagues travelled 9,589 kilometres from the Isle of Man, UK, to a region east of the Newfoundland shelf. The shark's nights were generally spent at depths of 200-300 metres and her days at 400-800 metres, once reaching 1,264 metres.

Correction
The Research Highlight 'Growing up bigger'
(Nature 453,567; 2008) said that deuterium
atoms in deuterated benzene can occupy more
space than hydrogen atoms in benzene owing to
vibrations of the carbon-deuterium and carbon-
hydrogen bonds. In fact, the effect is caused by
vibrations of the entire molecules about their
equilibrium positions.

\section{JOURNAL CLUB}

\section{Uri Alon \\ Weizmann Institute of Science, Rehovot, Israel}

\section{A biophysicist learns the art of hugging.}

If there are two things I love, they are warm hugs and simple answers to long-standing questions. Why must proteins bend in order to bind to their partners? This bending, known as induced fit, is puzzling, because it costs elastic energy and makes the binding less tight. But all sorts of proteins show induced fit such as antibodies that recognize viruses and regulatory proteins that embrace DNA - despite the fact that such processes would be more efficient if the protein and target fitted together like pieces of a jigsaw puzzle.

Enter two Israeli physicists, Tsvi Tlusty and Yonatan Savir. They used statistical mechanics to show that bending is a good idea if the goal is not to bind tightly but to avoid binding the wrong partner (Y. Savir and T. Tlusty PLoS ONE 2, e468; 2007).

Suppose that a protein needs to bind its target molecule ' $A$ ', and to avoid binding molecule ' $B$ ', which is a bit smaller than $A$ but otherwise similar in shape. The protein would do well to make its binding pocket a little larger than $A$; it would then have to bend a little to embrace $A$, at a small energy cost, but bend a lot to bind B. Crucially, the elastic energy required for a protein to flex rises ever more steeply with the extent of bending, just as that needed for a spring to bend rises with the square of bending. So the energy difference between binding $A$ and binding $B$ is greater with induced fit than it is when $A$ is a perfect fit.

Like all fruitful theories, this one makes testable predictions. It allows researchers to hypothesize what sort of imperfect fit might best serve a particular protein so that binding to non-target molecules is minimized. An antibody that attaches to virus proteins should be able to avoid similarly shaped human proteins, for instance. So perhaps, as with people, if you really want to know whether proteins are made for each other, it's in the hug.

Discuss this paper at http://blogs. nature.com/nature/journalclub 